\title{
New Measurement of the Capture Cross Section of Bismuth and Lead Isotopes
}

Cite as: AIP Conference Proceedings 769, 1521 (2005); https://doi.org/10.1063/1.1945294

Published Online: 13 June 2005

C. Domingo-Pardo, J. L. Tain, U. Abbondanno, G. Aerts, H. Álvarez, F. Alvarez-Velarde, S. Andriamonje, J. Andrzejewski, P. Assimakopoulos, L. Audouin, G. Badurek, P. Baumann, F. Bečvář, J. Benlliure, E. Berthoumieux, F. Calviño, D. Cano-Ott, R. Capote, A. Carrillo de Albornoz, P. Cennini, V. Chepel, E. Chiaveri, N. Colonna, G. Cortes, D. Cortina, A. Couture, J. Cox, S. David, R. Dolfini, W. Dridi, I. Duran, M. Embid-Segura, L. Ferrant, A. Ferrari, L. Fitzpatrick, R. Ferreira-Marques, H. Frais-Koelbl, K. Fujii, W. Furman, C. Guerrero, I. Goncalves, R. Gallino, E. Gonzalez-Romero, A. Goverdovski, F. Gramegna, E. Griesmayer, F. Gunsing, B. Haas, R. Haight, M. Heil, A. Herrera-Martinez, M. Igashira, S. Isaev, E. Jericha, Y. Kadi, F. Käppeler, D. Karamanis, D. Karadimos, M. Kerveno, V. Ketlerov, P. Koehler, V. Konovalov, E. Kossionides, M. Krtička, C. Lamboudis, H. Leeb, A. Lindote, I. Lopes, M. Lozano, S. Lukic, J. Marganiec, L. Marques, S. Marrone, P. Mastinu, A. Mengoni, P. M. Milazzo, C. Moreau, M. Mosconi, F. Neves, H. Oberhummer, S. O'Brien, M. Oshima, J. Pancin, C. Papachristodoulou, C. Papadopoulos, C. Paradela, N. Patronis, A. Pavlik, P. Pavlopoulos, L. Perrot, R. Plag, A. Plompen, A. Plukis, A. Poch, C. Pretel, J. Quesada, T. Rauscher, R. Reifarth, M. Rosetti, C. Rubbia, G. Rudolf, P. Rullhusen, J. Salgado, L. Sarchiapone, C. Stephan, G. Tagliente, L. Tassan-Got, L. Tavora, R. Terlizzi, G. Vannini, P. Vaz, A. Ventura, D. Villamarin, M. C. Vincente, V. Vlachoudis, R. Vlastou, F. Voss, H. Wendler, M. Wiescher, K. Wisshak, and The n_TOF Collaboration

\section{ARTICLES YOU MAY BE INTERESTED IN}

Measurements at $\mathrm{n}$ _TOF of the Neutron Capture Cross Section of Minor Actinides Relevant to the Nuclear Waste Transmutation

AIP Conference Proceedings 769, 1442 (2005); https://doi.org/10.1063/1.1945275

The n_TOF Facility at CERN: Performances and First Physics Results

AIP Conference Proceedings 769, 724 (2005); https://doi.org/10.1063/1.1945110

Measurement of the ${ }^{232}$ Th Neutron Capture Cross Section at the CERN n_TOF Facility AIP Conference Proceedings 769, 1470 (2005); https:// doi.org/10.1063/1.1945282
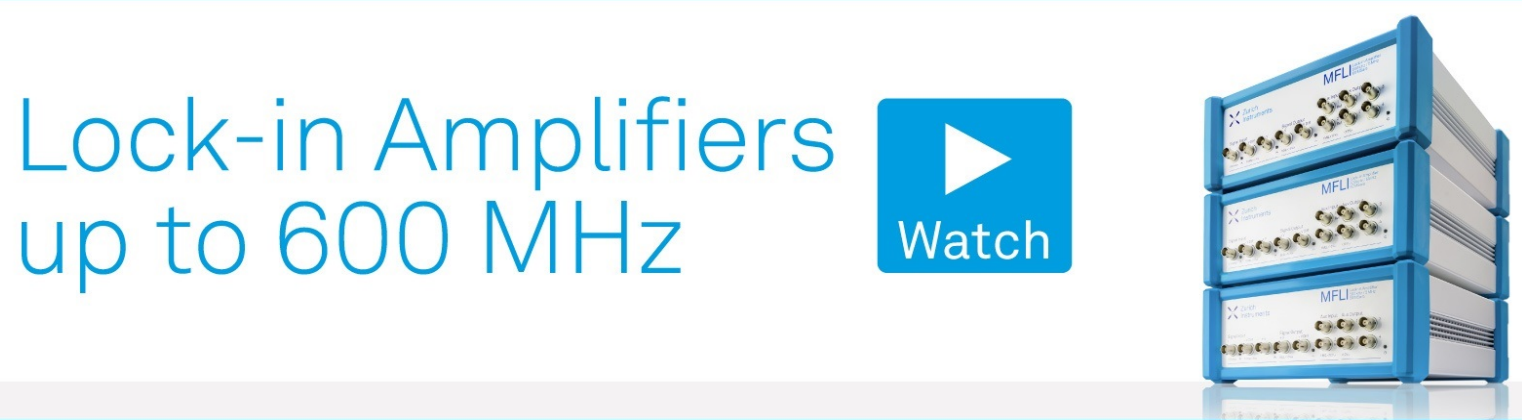


\title{
New Measurement of the Capture Cross Section of Bismuth and Lead Isotopes
}

\author{
C. Domingo-Pardo ${ }^{21}$, J.L. Tain ${ }^{21}$, U. Abbondanno ${ }^{14}$, G. Aerts ${ }^{7}$, H. Álvarez ${ }^{24}$, F. Alvarez-Velarde ${ }^{20}$,
}

S. Andriamonje ${ }^{7}$, J. Andrzejewski ${ }^{33}$, P. Assimakopoulos ${ }^{9}$, L. Audouin ${ }^{5}$ G. Badurek ${ }^{1}$, P. Baumann ${ }^{6}$, F. Bečvár ${ }^{31}$, J. Benlliure ${ }^{24}$, E. Berthoumieux ${ }^{7}$, F. Calviño ${ }^{25}$, D. Cano-Ott ${ }^{20}$, R. Capote ${ }^{23}$, A. Carrillo de Albornoz ${ }^{30}$, P. Cennini $^{4}$, V. Chepel ${ }^{17}$, E. Chiaveri ${ }^{4}$, N. Colonna ${ }^{13}$, G. Cortes $^{25}$, D. Cortina ${ }^{24}$, A. Couture ${ }^{29}$, J. Cox ${ }^{29}$, S. David $^{5}$, R. Dolfini ${ }^{15}$, W. Dridi ${ }^{7}$, I. Duran ${ }^{24}$, M. Embid-Segura ${ }^{20}$, L. Ferrant ${ }^{5}$, A. Ferrari ${ }^{4}$, L. Fitzpatrick ${ }^{4}$, R. Ferreira-Marques ${ }^{17}$, H. Frais-Koelbl ${ }^{3}$, K. Fujiii ${ }^{13}$ W. Furman ${ }^{18}$, C. Guerrero ${ }^{20}$ I. Goncalves $^{30}$, R. Gallino ${ }^{36}$, E. Gonzalez-Romero ${ }^{20}$, A. Goverdovski ${ }^{19}$, F. Gramegna ${ }^{12}$, E. Griesmayer ${ }^{3}$, F. Gunsing ${ }^{7}$, B. Haas ${ }^{32}$, R. Haight ${ }^{27}$, M. Heil ${ }^{8}$,

A. Herrera-Martinez ${ }^{4}$, M. Igashira ${ }^{37}$, S. Isaev ${ }^{5}$, E. Jericha ${ }^{1}$, Y. Kadi ${ }^{4}$, F. Käppeler ${ }^{8}$, D. Karamanis ${ }^{9}$, D. Karadimos ${ }^{9}$, M. Kerveno, ${ }^{6}$, V. Ketlerov ${ }^{19}$, P. Koehler ${ }^{28}$, V. Konovalov ${ }^{18}$, E. Kossionides ${ }^{39}$, M. Krtička ${ }^{31}$, C. Lamboudis ${ }^{10}$, H. Leeb ${ }^{1}$, A. Lindote ${ }^{17}$, I. Lopes ${ }^{17}$, M. Lozano ${ }^{23}$, S. Lukic ${ }^{6}$, J. Marganiec ${ }^{33}$, L. Marques ${ }^{30}$, S. Marrone ${ }^{13}$,

P. Mastinu ${ }^{12}$, A. Mengoni ${ }^{4}$, P.M. Milazzo ${ }^{14}$, C. Moreau ${ }^{14}$, M. Mosconi ${ }^{8}$, F. Neves ${ }^{17}$, H. Oberhummer ${ }^{1}$, S. O’ Brien $^{29}$,

M. Oshima ${ }^{38}$, J. Pancin 7 , C. Papachristodoulou ${ }^{9}$, C. Papadopoulos ${ }^{40}$, C. Paradela ${ }^{24}$, N. Patronis ${ }^{9}$, A. Pavlik ${ }^{2}$,

P. Pavlopoulos ${ }^{34}$, L. Perrot ${ }^{7}$, R. Plag ${ }^{8}$, A. Plompen ${ }^{16}$, A. Plukis ${ }^{7}$, A. Poch ${ }^{25}$, C. Pretel ${ }^{25}$, J. Quesada ${ }^{23}$, T. Rauscher $^{26}$, R. Reifarth ${ }^{27}$, M. Rosetti ${ }^{11}$, C. Rubbia ${ }^{15}$, G. Rudolf ${ }^{6}$, P. Rullhusen ${ }^{16}$, J. Salgado ${ }^{30}$, L. Sarchiapone ${ }^{4}$, C. Stephan ${ }^{5}$, G. Tagliente ${ }^{13}$, L. Tassan-Got ${ }^{5}$, L. Tavora ${ }^{30}$, R. Terlizzi ${ }^{13}$, G. Vannini ${ }^{35}$, P. Vaz ${ }^{30}$, A. Ventura ${ }^{11}$, D. Villamarin ${ }^{20}$, M. C. Vincente ${ }^{20}$, V. Vlachoudis ${ }^{4}$, R. Vlastou ${ }^{40}$ F. Voss $^{8}$, H. Wendler ${ }^{4}$, M. Wiescher ${ }^{29}$, K. Wisshak ${ }^{8}$ The n_TOF Collaboration

${ }^{1}$ Atominstitut der Österreichischen Universitäten, Technische Universität Wien, Austria $-{ }^{2}$ Institut für Isotopenforschung und Kernphysik, Universität Wien, Austria - ${ }^{3}$ Fachhochschule Wiener Neustadt, Wiener Neustadt, Austria - ${ }^{4}$ CERN, Geneva, Switzerland - ${ }^{5}$ Centre National de la Recherche Scientifique/IN2P3 IPN, Orsay, France - ${ }^{6}$ Centre National de la Recherche Scientifique/IN2P3 - IReS, Strasbourg, France -

${ }^{7}$ CEA/Saclay - DSM, Gif-sur-Yvette, France - ${ }^{8}$ Forschungszentrum Karlsruhe GmbH (FZK), Institut für Kernphysik, Germany - ${ }^{9}$ University of Ioannina, Greece - ${ }^{10}$ Aristotle University of Thessaloniki, Greece -

${ }^{11}$ ENEA, Bologna, Italy - ${ }^{12}$ Laboratori Nazionali di Legnaro, Italy $-{ }^{13}$ Istituto Nazionale di Fisica Nucleare, Bari, Italy - ${ }^{14}$ Istituto Nazionale di Fisica Nucleare, Trieste, Italy - ${ }^{15}$ Università degli Studi Pavia, Pavia, Italy $-{ }^{16}$ CEC-JRC-IRMM, Geel, Belgium - ${ }^{17}$ LIP - Coimbra \& Departamento de Fisica da Universidade de Coimbra, Portugal - ${ }^{18}$ Joint Institute for Nuclear Research, Frank Laboratory of Neutron Physics, Dubna, Russia - ${ }^{19}$ Institute of Physics and Power Engineering, Kaluga region, Obninsk, Russia - ${ }^{20}$ Centro de Investigaciones Energeticas Medioambientales y Technologicas, Madrid, Spain - ${ }^{21}$ Consejo Superior de Investigaciones Cientificas - University of Valencia, Spain - ${ }^{22}$ Universidad Politecnica de Madrid, Spain -

${ }^{23}$ Universidad de Sevilla, Spain - ${ }^{24}$ Universidade de Santiago de Compostela, Spain - ${ }^{25}$ Universitat Politecnica de Catalunya, Barcelona, Spain - ${ }^{26}$ Department of Physics and Astronomy - University of Basel, Basel, Switzerland - ${ }^{27}$ Los Alamos National Laboratory, New Mexico, USA - ${ }^{28}$ Oak Ridge National Laboratory, Physics Division, Oak Ridge, USA - ${ }^{29}$ University of Notre Dame, Notre Dame, USA - ${ }^{30}$ Instituto Tecnológico e Nuclear, Lisbon, Portugal $-{ }^{31}$ Charles University, Prague, Czech Republic $-{ }^{32}$ Centre National de la Recherche Scientifique/IN2P3 - CENBG, Bordeaux, France - ${ }^{33}$ University of Lodz, Lodz, Poland - ${ }^{34}$ Pôle

Universitaire Léonard de Vinci, Paris La Défense, France - ${ }^{35}$ Dipartimento di Fisica, Università di Bologna, and Sezione INFN di Bologna, Italy - ${ }^{36}$ Dipartimento di Fisica Generale, Università di Torino and Sezione

INFN di Torino, I-10125 Torino, Italy - ${ }^{37}$ Tokyo Institute of Technology, Tokyo, Japan - ${ }^{38}$ Japan Atomic Energy Research Institute, Tokai-mura, Japan $-{ }^{39}$ NCSR, Athens, Greece - ${ }^{40}$ National Technical University of Athens, Greece

Abstract. All the stable lead isotopes and ${ }^{209} \mathrm{Bi}$ have been recently measured at $\mathrm{n}_{-}$TOF [1] (CERN) in the range from $1 \mathrm{eV}$ up to $1 \mathrm{MeV}$, using an optimized experimental setup. A set of two homemade $\mathrm{C}_{6} \mathrm{D}_{6}$ detectors with carbon-fibre canning was designed to greatly reduce the sensitivity to scattered neutrons. Surrounding elements like the sample holder and the sample exchanger have been made also of carbon fibre.

The pulse-height weighting technique has been used in order to determine the $(\mathrm{n}, \gamma)$ cross sections. Concerning this technique, special care has been taken in the treatment of several sources of error leading to a total systematic uncertainty of less than $3 \%$.

Results on the analysed ${ }^{209} \mathrm{Bi}$ and ${ }^{207} \mathrm{~Pb}$ capture data and a comparison with previous experiments and evaluations are presented.

CP769, International Conference on Nuclear Data for Science and Technology, edited by R. C. Haight, M. B. Chadwick, T. Kawano, and P. Talou (C) 2005 American Institute of Physics 0-7354-0254-X/05/\$22.50 


\section{INTRODUCTION}

Lead and bismuth capture data are important in the field of energy production and nuclear-waste transmutation, in order to evaluate the neutron balance in the core of an Accelerator Driven System (ADS) with a $\mathrm{Pb}-\mathrm{Bi}$ spallation target and also to account for the synthesis of ${ }^{210 \mathrm{~m}} \mathrm{Bi}$ through capture reactions, which determines the longterm radioactivity of the spallation source.

In astrophysics, ${ }^{208} \mathrm{~Pb}$ and ${ }^{209} \mathrm{Bi}$ represent the end point of the s-process, which by means of $(n, \gamma)$ reactions builds around half of the isotopic abundances in the mass region $\mathrm{A} \geq 56$. Accurate capture data on these end-point isotopes are required to further develop nucleosynthesis stellar models and obtain heavy-element abundance predictions compatible with observations.

Capture in these isotopes is difficult to measure not only due to their small cross sections but also due to the relatively high rate of scattered neutrons that can interact in the detector and surrounding material and mimic a capture signal. This has been a source of systematic uncertainty in previous experiments.

\section{EXPERIMENT}

Neutron-energy differential capture cross sections have been determined with high resolution at $\mathrm{n}$ _TOF thanks to the 185-m-long flight path and the initial sharp pulsed, $6 \mathrm{~ns}$ wide (RMS), $20 \mathrm{GeV} / \mathrm{c}$ proton beam that produces the neutrons by spallation on a lead block.

The key improvement with respect to previous experiments is a very low neutron sensitivity, achieved mainly by means of innovative carbon-fibre homemade $\mathrm{C}_{6} \mathrm{D}_{6}$ detectors [2]. Other elements, like the sample holder and the sample exchanger, are made also from carbon fibre. Such a detection setup has been specially conceived for the accurate measurement of nuclides with resonances showing large scattering to capture ratios, which is in general the case of the lead and bismuth isotopes.

Two carbon-fibre $\mathrm{C}_{6} \mathrm{D}_{6}$ detectors were placed backwards at $\sim 135^{\circ}$ in order to minimize the effects of the primary angular distribution in the case of ${ }^{207} \mathrm{~Pb}$ but also to reduce detection of in-beam $\gamma$-rays, which are scattered on the sample and constitute, in these particular measurements, the main source of background.

The neutron flux was monitored in parallel to the capture measurements by centering a thin ${ }^{6} \mathrm{Li}$ foil in the beam a few meters before the capture sample under study. The outgoing ${ }^{3} \mathrm{H}$ particles from the ${ }^{6} \mathrm{Li}(\mathrm{n}, \alpha)$ reaction were registered by means of four $\mathrm{Si}$-detectors placed off the beam but covering a high solid angle.

\footnotetext{
1 corresponding author: cesar.domingo.pardo@cern.ch
}

The cross section was measured relative to the standard 4.9-eV ${ }^{197} \mathrm{Au}$ resonance; therefore a thin Au-sample was regularly measured during the experiment. To carry out this normalization, the dependence of the neutron flux against the neutron energy is also needed. This has been determined not only with the Si-detectors mentioned above, but also from dedicated ${ }^{235,238} \mathrm{U}$ fission measurements. The flux has been finally evaluated from both measurements, and its associated uncertainty can be considered on average below $1 \%$ for neutron energies up to $100 \mathrm{keV}$ and increases up to $3 \%$ at $500 \mathrm{keV}$.

The $(\mathrm{n}, \gamma)$ cross section has been measured using the Pulse Height Weighting Technique (PHWT). This is based on the appropriate manipulation of the detector response in order to achieve a detection efficiency independent of the nuclear $\gamma$-cascade. As has been shown in a previous work [4], the Weighting Function depends on the exact $(n, \gamma)$ detection setup, including the sample itself. Therefore, a complete and realistic Monte Carlo simulation represents the only reliable method to determine it accurately.
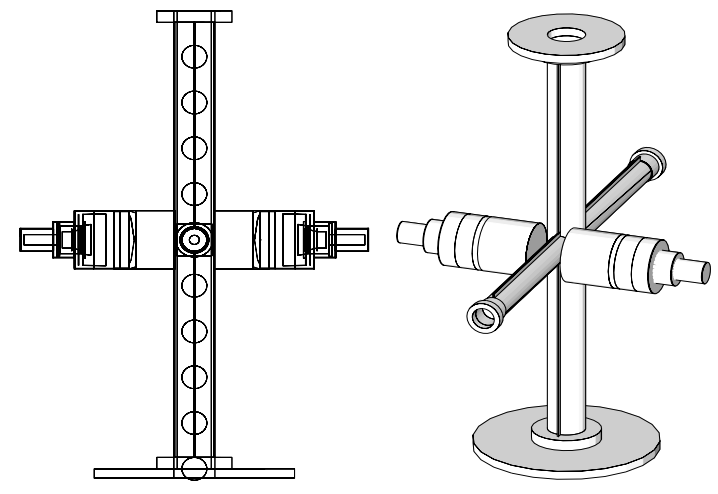

FIGURE 1. View of the $(\mathrm{n}, \gamma)$ detection setup as is implemented in the code GEANT4[5] used for the MC simulations.

Before starting with the measurement of the isotopes of interest, part of the n_TOF beam time was dedicated to validate and determine the systematic accuracy of the PHWT [3]. The $1.15-\mathrm{keV}^{56} \mathrm{Fe}$ resonance constitutes an ideal accuracy test for a radiative neutron-capture measurement. This resonance is strong and well isolated, and its capture width $\Gamma_{\gamma}$ is about ten times larger than its neutron width $\Gamma_{n}$, thus becoming the radiative-capture measurement mainly sensitive to $\Gamma_{n}$.

An exhaustive modelling of the measuring setup in the MC simulation (see Fig. 1), together with a detailed analysis of the different sources of systematic uncertainty like electronic threshold, summing of two or more $\gamma$ rays, and Conversion Electrons (CE), enabled us to determine the $1.15-\mathrm{keV}^{56} \mathrm{Fe}$ resonance cross section with an accuracy better than $2 \%$, which is then a good measure of our experimental-technique systematic uncertainty. 


\section{ANALYSIS}

The present work describes the results obtained for ${ }^{209} \mathrm{Bi}$ and ${ }^{207} \mathrm{~Pb}$. In the case of ${ }^{209} \mathrm{Bi}$, apart from the common experimental sources of systematical uncertainty mentioned above (threshold, $\gamma$-summing, and CE), there exists an additional effect, which is the isomeric state at $270 \mathrm{keV}$. In a similar way as in the case of the 1.15$\mathrm{keV}{ }^{56} \mathrm{Fe}$ resonance described in [3], we estimated all these effects utilizing the statistical model of the nucleus. A satisfactory description of the deposited energy spectrum was obtained when compared with the experimental spectrum, as shown in the left part of Fig. 2. In ${ }^{207} \mathrm{~Pb}$,
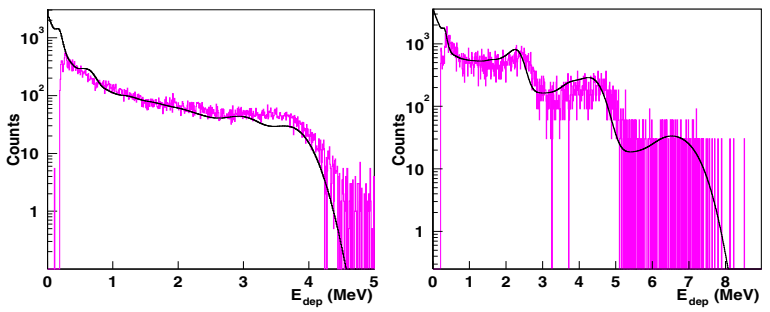

FIGURE 2. Left: MC simulation (black line) of the pulseheight spectrum for the $802-\mathrm{eV}^{209} \mathrm{Bi}$ resonance and comparison with the experimental spectrum. Right: simulation of the deposited energy spectrum for the $3-\mathrm{keV} 2^{+}$resonance in ${ }^{207} \mathrm{~Pb}$ and comparison with the experimental spectrum.

capture of one neutron leads to the double magic ${ }^{208} \mathrm{~Pb}$, which shows a very simple deexcitation pattern of only one or two transitions to the ground state depending on the spin and parity of the initial level. Therefore, the response distribution was reproduced by including those transitions in the MC simulation rather than using the statistical model. The resulting deposited energy spectrum shows good agreement with the corresponding experimental one as shown in the right part of Fig. 2.

For a $\gamma$-threshold of $200 \mathrm{keV}$, the calculated yieldcorrection factors are 1.046(4), 1.137(4), and 1.016(4), respectively, for the $4.9-\mathrm{eV}^{197} \mathrm{Au}, 802-\mathrm{eV}^{209} \mathrm{Bi}$, and the $3-\mathrm{keV}^{207} \mathrm{~Pb}$ resonances. It is important to note here that an accurate determination of the final capture yield can only be made after correcting for these effects, particularly in the case of bismuth and gold, where the corrections are larger.

The neutron-sensitivity correction was negligible (below $0.7 \%$ ) for all the analysed resonances except for the 12.1-keV level of ${ }^{209} \mathrm{Bi}$. In this case the ratio $\Gamma_{n} / \Gamma_{\gamma} \sim$ $10^{3}$ required a correction of $1.5 \%$.

The experimental yield, corrected for these effects, was analysed using the multilevel $R$-matrix code SAMMY [6]. The cross sections were parameterized using the Reich-Moore formalism and the yields calculated for a given sample composition and thickness. The main broadening in the keV region, which is due to the atomic thermal motion, is calculated from the temperature. Sample self-shielding, single-, and doubleneutron scattering in the sample are also accounted for by this code. In addition, the broadening due to the neutron-beam resolution function has been included by means of the SAMMY-RPI parameterization. When a good transmission measurement existed for the neutron width, $\Gamma_{n}$, this parameter was kept fixed together with the rest of "a priori" information mentioned above and the value of $\Gamma_{\gamma}$ was derived by SAMMY using the Bayes' theorem. In case the resonance has been only observed in the capture measurement, then only the value of the radiative kernel or capture area was derived usually by fitting both $\Gamma_{n}$ and $\Gamma_{\gamma}$.

Due to the limited statistics available, we observed 21 resonances in the case of ${ }^{209} \mathrm{Bi}$ in the energy region between 0.8 and $23 \mathrm{keV}$. In ${ }^{207} \mathrm{~Pb}$ the number of observed resonances was 19 in the region from 3 to $317 \mathrm{keV}$. Despite the backwards position of both $\mathrm{C}_{6} \mathrm{D}_{6}$ detectors, the in-beam $\gamma$-rays were still the main source of background, hiding most of the weaker resonances. The situation should improve very much in the future with the eventual replacement of the water moderator of the spallation target by heavy water. In this case, the in-beam $\gamma$-rays will be highly suppressed and the associated background should become practically negligible.

In Fig. 3, fit examples are given for the two isotopes presented in this work.
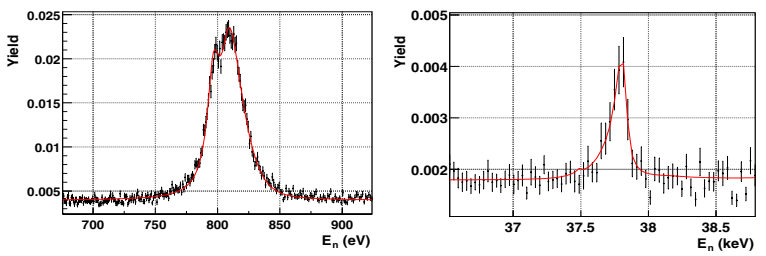

FIGURE 3. SAMMY fit of the $s$-wave ${ }^{209} \mathrm{Bi}$ resonance at $802 \mathrm{eV}$ (left) and the ${ }^{207} \mathrm{~Pb}$ resonance at $37 \mathrm{keV}$ (right).

\section{RESULTS}

In the case of ${ }^{209} \mathrm{Bi}$, most of the resonances are found in good agreement with the previous measurement performed at Geel [9] (see Fig. 4). Nevertheless, the systematic deviation for $s$-wave resonances at lower energy indicates a small overcorrection of their neutron sensitivity. It is worth noting in this case the large discrepancies found with the evaluated data files, which are based on the first capture experiment of this isotope performed at Oak Ridge [10]. This shows larger capture areas, mainly for $s$-wave resonances, due to the higher neutron sensitivity of their experimental setup, which required corrections as large as $\sim 50 \%$, with correspondingly large uncertainties. 


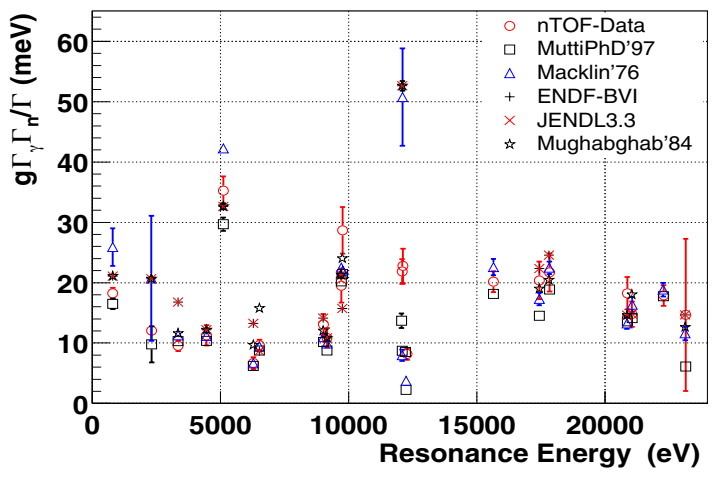

FIGURE 4. Comparison of the Bi radiative kernel measured at $\mathrm{n} \_\mathrm{TOF}$ versus previous experiments and evaluated files.

In the case of ${ }^{207} \mathrm{~Pb}$, previous measurements of the resonance parameters or capture areas have been compared with n_TOF $[11,12,13,14]$ (Fig. 5). The capture areas calculated from the evaluations JENDL and ENDF and those from the compilations $[7,8]$ have been also included. Beyond $100 \mathrm{keV}$, again large discrepancies are

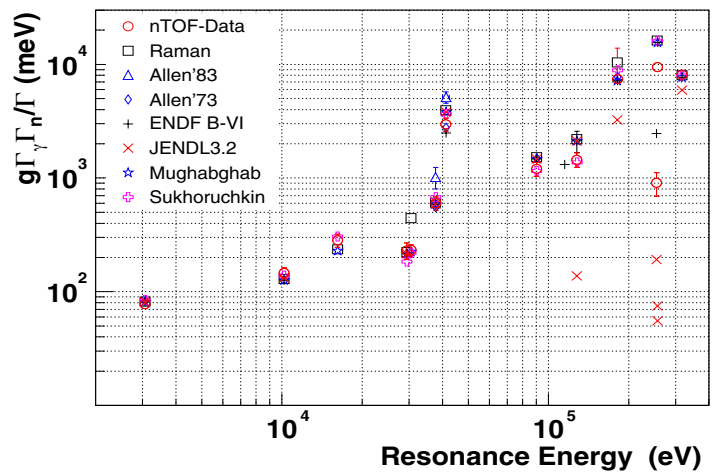

FIGURE 5. Radiative kernels measured at $n \_T O F$ for ${ }^{207} \mathrm{~Pb}$ versus previous measurements and evaluated files.

found with the evaluations. In the rest of the cases the agreement is satisfactory for almost every resonance.

According to Figs. 4 and 5, larger discrepancies have been found in the first case $\left({ }^{209} \mathrm{Bi}\right)$. Thus it is interesting to discuss its impact on the calculations for stellar nucleosynthesis and abundances. The relevant quantity for that calculation is the Maxwellian Averaged Cross Section (MACS). It determines the survival of ${ }^{209} \mathrm{Bi}$ and the production of the unstable ${ }^{210} \mathrm{Bi}$ that $\alpha$-decays, contributing to the production of ${ }^{206} \mathrm{~Pb}$. In Fig. 6, the ${ }^{209} \mathrm{Bi}$ MACS obtained for the capture areas measured at $n \_$TOF is compared with that obtained from the two previous experiments at Geel and Oak Ridge. The effect of these new results should be checked by including them in an appropriate stellar code.

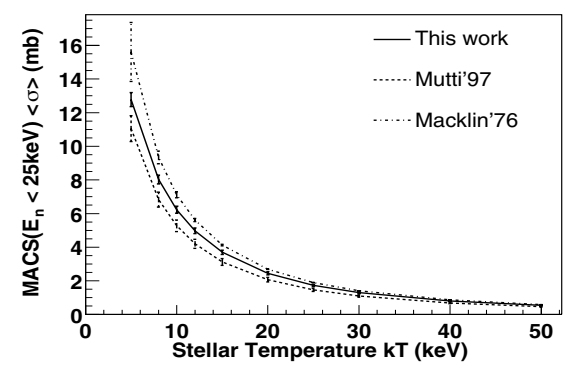

FIGURE 6. Maxwellian-averaged cross section versus the stellar temperature calculated for neutron-energy resonances below $25 \mathrm{keV}$.

Another open question comes from the calculation of the thermal-capture cross section as the sum of the tails of the $s$-wave resonances. Computing it for the latest experiments (Geel and $\mathrm{n}_{-} \mathrm{TOF}$ ) one obtains a value of $\sigma_{t h} \approx 24 \mathrm{mb}$, which is about $40 \%$ lower than the adopted value of 33.8(7) $\mathrm{mb}$ [7]. Whether this might be due to subthreshold resonances or a direct-capture component remains to be investigated.

\section{CONCLUSIONS}

High-resolution measurements on ${ }^{204,206,207,208} \mathrm{~Pb}$ and ${ }^{209} \mathrm{Bi}$ have been performed at the CERN n_TOF facility. The results on ${ }^{207} \mathrm{~Pb}$ and ${ }^{209} \mathrm{Bi}$ reported in this work reflect the low-neutron sensitivity achieved thanks to the very low-mass detection setup and elements. A systematic accuracy better than $3 \%$ was achieved by exhaustive treatment of the different sources of systematic uncertainties. The two figures comparing the radiative kernels clearly suggest updating the information existing in the evaluated libraries.

\section{REFERENCES}

1. The n_TOF Collaboration, CERN/SPSC 99-8, SPSC/P 310.

2. R. Plag et al., Nuc. Instrum. Methods A 496 (2003) 425-436.

3. U. Abbondanno et al., Nucl. Instrum. Methods A, 521 (2004) 454-467.

4. J.L. Tain et al., J. Nucl. Sci. Technol., Sup. 2 (2002), p. 689.

5. S. Agostinelli et al., Nucl. Instrum. Methods A, 506, (2003), p. $250-303$.

6. N.M. Larson, ORNL/TM-9179, Oak Ridge NL, 2000.

7. S.F.Mughabghab, Neutron Cross Sections Academic press, 1984.

8. S.I. Sukhoruchkin et al., Low En. Neutron Physics, Springer, 1998.

9. P. Mutti, PhD Thesis, University of Gent, 1997.

10. R. Macklin and J. Halperin, Phys. Rev. C, 14-4, 1976.

11. B.J. Allen et al., Phys. Rev. C, 8-4, 1973.

12. B.J. Allen et al., J. Phys. G. 1173, 1980.

13. S. Raman et al., Phys. Rev. Let., 39-10, 1977.

14. S. Raman et al., Phys. Rev. Let., 40-20, 1978. 